\title{
Editorial on: "Characteristics of extension and de novo recurrent spontaneous coronary artery dissection"
}

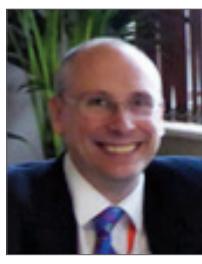

David Adlam*, DPhil, FRCP; Alice Wood, MBBS, MRCP; Abtehale Al-Hussaini, MBBS, MRCP

Department of Cardiovascular Sciences, University of Leicester, Glenfield Hospital, Leicester, United Kingdom

Spontaneous coronary artery dissection (SCAD), although uncommon, remains an under-recognised cause of acute myocardial infarction (AMI), afflicting predominantly (but not exclusively) young to middle-aged women, including a minority $(\sim 10 \%)$ of cases occurring peripartum and postpartum ${ }^{1-3}$. The condition results from the external compression of a coronary artery by the development of a false lumen in the outer third of the tunica media of the coronary arterial wall (Figure 1). This causes impairment of blood flow in the true lumen leading to myocardial ischaemia and infarction.

Once limited to anecdotal case reports, the understanding of SCAD has advanced significantly in recent years as a result of data from larger retrospective registries ${ }^{1-3}$. Although the underlying pathophysiological mechanisms remain unknown, these studies have demonstrated an important association between SCAD and the presence of extracoronary abnormalities (especially fibromuscular dysplasia $[\mathrm{FMD}])^{4,5}$, suggesting that this condition may be a manifestation of a more generalised arteriopathy.

Clinically, these studies have confirmed the challenges of revascularisation in $\mathrm{SCAD}^{6-10}$. Outcomes from percutaneous coronary intervention (PCI) are worse than in atherosclerotic disease, predominantly due to the risk of axial migration of the haematoma during coronary dilation and stenting. Coronary artery bypass grafting $(\mathrm{CABG})$ is generally reserved as a bail-out option either for failed PCI or for very high-risk cases with extensive proximal dissection, although long-term graft patency rates appear reduced $^{10}$. Importantly, unlike in atherosclerotic AMI, a conservative approach to revascularisation is generally favoured in cases where there is maintained coronary flow and no evidence of significant ongoing ischaemia or infarction. In the overwhelming majority of conservatively managed cases, there is complete resorption of the false lumen with full restoration of normal coronary architecture by three to six months post $\mathrm{SCAD}^{7-10}$. Optimal medical therapy both in the acute context and after SCAD remains to be clearly elucidated, although one recent series has provided the first data suggesting beta-blockade may have a role ${ }^{11}$.

In the study by Main et al from Vancouver reported in this issue of EuroIntervention ${ }^{12}$, the issue of SCAD recurrence is explored.

\section{Article, see page 1454}

Importantly, the authors separate out recurrent de novo SCAD from extension of an existing dissection and other causes of major adverse cardiovascular events (MACE), such as stent thrombosis and restenosis. This paper makes important new contributions to our understanding of SCAD outcomes.

*Corresponding author: Department of Cardiovascular Sciences, University of Leicester, Glenfield Hospital, Groby Road, 


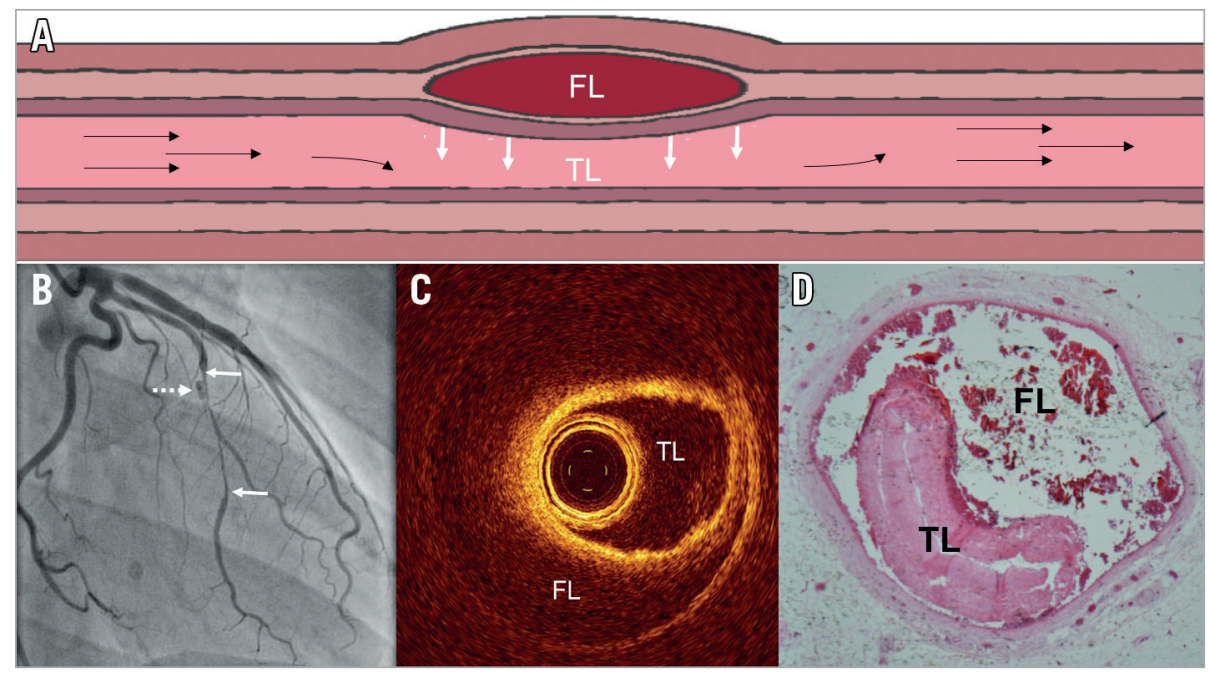

Figure 1. Anatomy of SCAD. A) Schematic diagram of SCAD. Accumulation of blood within the outer third of the tunica media forms a false lumen (FL) leading to external compression of the true lumen (TL) and obstruction of blood flow. B) Angiographic appearance of SCAD leading to a long stenosis (solid white arrows) and localised contrast penetration of the false lumen (dotted arrow). OCT (C) and histopathological (D) appearances of SCAD.

Extension of an existing dissection following an index SCAD event appears rare. In this series, just nine such cases (from $310-$ $2.9 \%$ ) are reported and only five of these involved extensions beyond the initially affected coronary segment. This low reported rate of dissection extension would appear to provide further support to the growing consensus favouring a conservative revascularisation strategy where possible for $\mathrm{SCAD}^{1-3}$. Clearly some cases in the series will have been managed with PCI (where extension post procedure is unlikely), so these extension events should ideally be interpreted as a proportion of those cases managed conservatively. Whilst the revascularisation strategy adopted in the overall cohort is not reported in this manuscript, previous publications from the same group report that a majority of their cases $(83.1 \%)$ are managed conservatively ${ }^{11}$, supporting the assertion that, in conservatively managed SCAD, extension of dissection is uncommon. Although unusual, a minority of extension events appear significant, with $3 / 9$ presenting with ST elevation; 6/9 however were still able to continue on a conservative revascularisation strategy. Importantly, the median time to extension was five days with events reported up to 19 days. Although the numbers are small, this provides a note of caution regarding early hospital discharge after conservatively managed SCAD and hints at the potential importance of appropriate counselling of patients when they do leave hospital.

De novo recurrence is a major concern for patients surviving SCAD and prevention of these events is a key target for therapeutic interventions. The first retrospective series from the Mayo Clinic in the USA reported 10-year recurrence rates as high as $29.4 \%{ }^{13}$, although this figure was potentially exaggerated by a degree of inherent selection bias. In the paper by Main et al, an overall incidence of de novo recurrence of $11 \%$ from 310 patients is described. Although the median follow-up is not reported, the same Vancouver group has recently published a rate of recurrent de novo SCAD of $10.4 \%$ from 327 patients in their cohort followed prospectively for a median 3.1 years ${ }^{11}$. This suggests that recurrence is indeed a significant risk for SCAD survivors. Interestingly, the authors report that recurrent events universally affected new coronary segments. They hypothesise that healed coronary segments may be more resistant to recurrent dissection. Whether or not this is true, the finding certainly strongly suggests that the arterial vulnerability in SCAD is not localised to a particular coronary segment but is part of a generalised arteriopathy. This is also in keeping with the high reported rates of FMD (74.4\%). Furthermore, this adds additional evidence to previous findings that PCI with stenting does not protect against SCAD recurrence, as late events usually affect different coronary territories $^{7,10}$. Importantly, the authors also report that most recurrent SCAD in this series was managed conservatively and outcomes were good, with no fatalities and relative preservation of left ventricular systolic function. So, beyond the need for revascularisation in some cases, short-term outcomes after recurrent events appear good, although the psychological morbidity (not reported here) may be significant and long-term outcomes in this young population remain to be elucidated.

Six patients (17.6\% of those with a first de novo recurrent SCAD) went on to have a second recurrence. Although the numbers are small, given the inherently shorter follow-up, this raises the question as to whether recurrence per se marks out a population at particularly high risk of further events. If confirmed in other series, this could have both clinical and pathophysiological significance.

SCAD remains a clinical challenge both acutely in the cardiac catheterisation laboratory and subsequently in the clinic. 
Whilst the findings reported by Main et al require corroboration in other large series, understanding and ultimately reducing the risk of dissection extension and de novo recurrence after SCAD are key to reducing patient morbidity. Further progress will require both larger prospective series with longer follow-up and international research collaboration targeting enhanced understanding of the underlying pathophysiology and ultimately the refinement of a specific treatment strategy for SCAD.

\section{Acknowledgements}

The authors are grateful for the support of the British Heart Foundation, the Leicester NIHR Biomedical Research Centre, the NIHR RD-TRC and Beat SCAD.

\section{Conflict of interest statement}

D. Adlam has received research funding from AstraZeneca and St. Jude Medical (now part of Abbott Vascular). The other authors have no conflicts of interest to declare.

\section{References}

1. Al-Hussaini A, Adlam D. Spontaneous coronary artery dissection. Heart. 2017;103:1043-51.

2. Saw J, Mancini GB, Humphries KH. Contemporary Review on Spontaneous Coronary Artery Dissection. J Am Coll Cardiol. 2016;68:297-312.

3. Tweet MS, Gulati R, Hayes SN. Spontaneous Coronary Artery Dissection. Curr Cardiol Rep. 2016;18:60.

4. Saw J, Poulter R, Fung A, Wood D, Hamburger J, Buller CE. Spontaneous coronary artery dissection in patients with fibromuscular dysplasia: a case series. Circ Cardiovasc Interv. 2012;5:134-7.

5. Toggweiler S, Puck M, Thalhammer C, Manka R, Wyss M, Bilecen D, Corti R, Amann-Vesti BR, Luscher TF, Wyss CA. Associated vascular lesions in patients with spontaneous coronary artery dissection. Swiss Med Wkly. 2012;142:w13538.

6. Lettieri C, Zavalloni D, Rossini R, Morici N, Ettori F, Leonzi O, Latib A, Ferlini M, Trabattoni D, Colombo P, Galli M, Tarantini G, Napodano M, Piccaluga E, Passamonti E, Sganzerla P, Ielasi A,
Coccato M, Martinoni A, Musumeci G, Zanini R, Castiglioni B. Management and Long-Term Prognosis of Spontaneous Coronary Artery Dissection. Am J Cardiol. 2015;116:66-73.

7. Nakashima T, Noguchi T, Haruta S, Yamamoto Y, Oshima S, Nakao K, Taniguchi Y, Yamaguchi J, Tsuchihashi K, Seki A, Kawasaki T, Uchida T, Omura N, Kikuchi M, Kimura K, Ogawa H, Miyazaki S, Yasuda S. Prognostic impact of spontaneous coronary artery dissection in young female patients with acute myocardial infarction: A report from the Angina Pectoris-Myocardial Infarction Multicenter Investigators in Japan. Int J Cardiol. 2016; 207:341-8.

8. Rogowski S, Maeder MT, Weilenmann D, Haager PK, Ammann P, Rohner F, Joerg L, Rickli H. Spontaneous Coronary Artery Dissection: Angiographic Follow-Up and Long-Term Clinical Outcome in a Predominantly Medically Treated Population. Catheter Cardiovasc Interv. 2017;89:59-68.

9. Saw J, Aymong E, Sedlak T, Buller CE, Starovoytov A, Ricci D, Robinson S, Vuurmans T, Gao M, Humphries K, Mancini GB. Spontaneous coronary artery dissection: association with predisposing arteriopathies and precipitating stressors and cardiovascular outcomes. Circ Cardiovasc Interv. 2014;7:645-55.

10. Tweet MS, Eleid MF, Best PJ, Lennon RJ, Lerman A, Rihal CS, Holmes DR Jr, Hayes SN, Gulati R. Spontaneous coronary artery dissection: revascularization versus conservative therapy. Circ Cardiovasc Interv. 2014;7:777-86.

11. Saw J, Humphries K, Aymong E, Sedlak T, Prakash R, Starovoytov A, Mancini GBJ. Spontaneous Coronary Artery Dissection: Clinical Outcomes and Risk of Recurrence. J Am Coll Cardiol. 2017;70:1148-58.

12. Main A, Prakash R, Starovoytov A, Sabbaghan A, Aymong E, Mancini GB, Saw J. Characteristics of extension and de novo recurrent spontaneous coronary artery dissection. EuroIntervention. 2017;13:e1454-59.

13. Tweet MS, Hayes SN, Pitta SR, Simari RD, Lerman A, Lennon RJ, Gersh BJ, Khambatta S, Best PJ, Rihal CS, Gulati R. Clinical features, management, and prognosis of spontaneous coronary artery dissection. Circulation. 2012;126:579-88. 